Swarthmore College

Works

$5-1-2002$

\title{
Energetic Particles From Three-Dimensional Magnetic Reconnection Events In The Swarthmore Spheromak Experiment
}

Michael R. Brown

Swarthmore College, doc@swarthmore.edu

C. D. Cothran

Matthew J. Landreman , '03

David J. Schlossberg , '01

Follow this and additional works at: https://works.swarthmore.edu/fac-physics

Part of the Physics Commons

Let us know how access to these works benefits you

\section{Recommended Citation}

Michael R. Brown; C. D. Cothran; Matthew J. Landreman , '03; and David J. Schlossberg , '01. (2002).

"Energetic Particles From Three-Dimensional Magnetic Reconnection Events In The Swarthmore Spheromak Experiment". Physics Of Plasmas. Volume 9, Issue 5. 2077-2084. DOI: 10.1063/1.1458589 https://works.swarthmore.edu/fac-physics/290

This work is brought to you for free by Swarthmore College Libraries' Works. It has been accepted for inclusion in Physics \& Astronomy Faculty Works by an authorized administrator of Works. For more information, please contact myworks@swarthmore.edu. 
Energetic particles from three-dimensional magnetic reconnection events in the Swarthmore Spheromak Experiment

M. R. Brown, C. D. Cothran, M. Landreman, and D. SchlossbergW. H. Matthaeus and G. QinV. S. Lukin and T. Gray

Citation: Physics of Plasmas 9, 2077 (2002); doi: 10.1063/1.1458589

View online: http://dx.doi.org/10.1063/1.1458589

View Table of Contents: http://aip.scitation.org/toc/php/9/5

Published by the American Institute of Physics

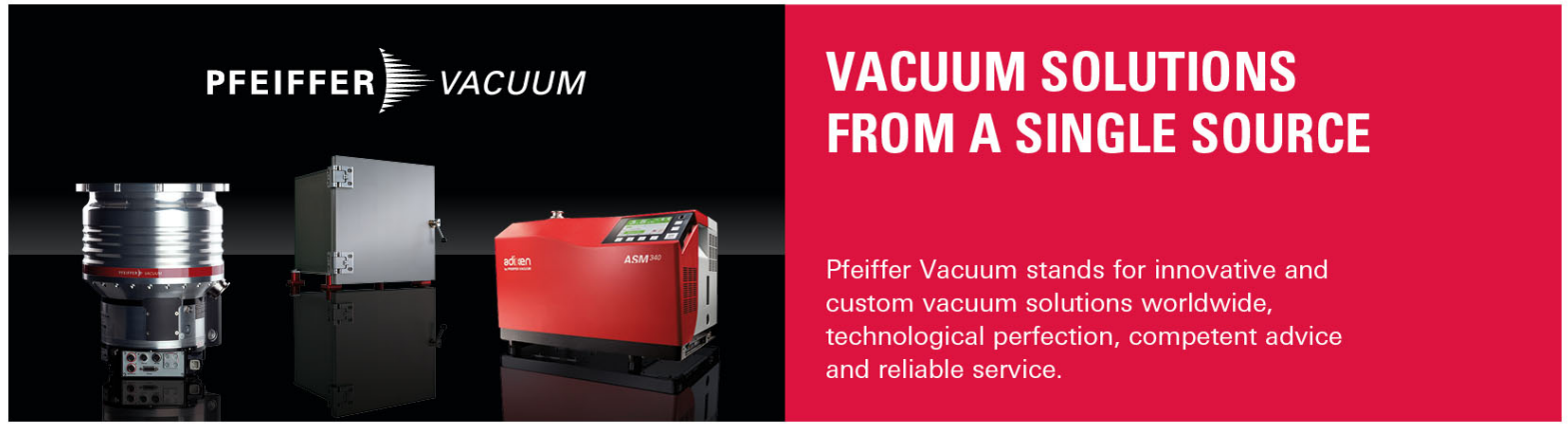




\title{
Energetic particles from three-dimensional magnetic reconnection events in the Swarthmore Spheromak Experiment ${ }^{a)}$
}

\author{
M. R. Brown, ${ }^{\text {b) }}$ C. D. Cothran, M. Landreman, and D. Schlossberg \\ Department of Physics and Astronomy, Swarthmore College, Swarthmore, Pennsylvania 19081-1397 \\ W. H. Matthaeus and G. Qin \\ Bartol Research Institute, University of Delaware, Newark, Delaware 19716 \\ V. S. Lukin and T. Gray \\ Princeton Plasma Physics Laboratory, Princeton University, Princeton, New Jersey 08543
}

(Received 30 October 2001; accepted 11 January 2002)

\begin{abstract}
Measurements are presented from the Swarthmore Spheromak Experiment (SSX) [M. R. Brown, Phys. Plasmas 6, 1717 (1999)] showing a population of superthermal, super-Alfvénic ions with $\bar{E}$ $\cong 90 \mathrm{eV}$ and $E_{\max } \geqslant 200 \mathrm{eV}$ accelerated by reconnection activity in three-dimensional magnetic structures. These energetic ions are temporally and spatially correlated with three-dimensional magnetic reconnection events (measured with a 3D probe array) and are accelerated along the X-line normal to the local 2D plane of reconnection. In a typical SSX discharge, the peak reconnection electromotive force $\mathcal{E}=v B L \leqslant\left(10^{5} \mathrm{~m} / \mathrm{s}\right)(0.05 \mathrm{~T})(0.1 \mathrm{~m})=500 \mathrm{~V}$ consistent with our observations. In addition, test particle simulations using magnetohydrodynamic (MHD) data from SSX simulations and run with dimensionless parameters similar to the experiment $(S=1000, \beta=0.1)$ show acceleration of ions up to $2 v_{\text {Alf }}$ in a few Alfvén times consistent with the measurement. The process includes two phases - a strong but short duration direct acceleration in the quasi-steady reconnection electric field, and a weaker longer lived sub-diffusive component associated with turbulence. (C) 2002 American Institute of Physics. [DOI: 10.1063/1.1458589]
\end{abstract}

\section{INTRODUCTION}

Magnetic reconnection is the process in which magnetic topology is globally rearranged due to local annihilation of magnetic flux or when magnetic fields cross-link. The paradigm for magnetic reconnection is the merger of two parcels of highly conductive magnetofluid with anti-parallel flux. ${ }^{1}$ High conductivity implies that the magnetic field and fluid are coupled (forming a magnetofluid). Magnetic reconnection converts magnetic energy to bulk flow, individual particle acceleration and heat. In two dimensions, the picture reduces to an inflow of magnetofluid, a decoupling of field and fluid by reconnection then flow of fluid across a separatrix (defined as those special field lines that cross at an $\mathrm{X}$-point) (see Fig. 1). By conservation of energy, the outflow velocity across the reconnection plane is limited to the Alfvén speed $v_{A}$.

In two dimensions, there is an electric field equal to the rate of change of magnetic flux at the X-point $(E$ $=d \psi /\left.d t\right|_{x}$ ). This electric field (essentially the reconnection rate) can accelerate ions along the $\mathrm{X}$-line. However, since the electric field is in the $\mathbf{v} \times \mathbf{B}$ direction, $\mathbf{E} \cdot \mathbf{B} \equiv 0$ identically. Ions that remain precisely on the $\mathbf{X}$-line (where $\mathbf{B} \equiv 0$ ) can be accelerated appreciably. However relatively few ions will participate in this acceleration unless some additional features such as multiple-X-lines, current sheets or turbulent

\footnotetext{
${ }^{a)}$ Paper LI2 3, Bull. Am. Phys. Soc. 46, 209 (2001).

${ }^{b)}$ Invited speaker.
}

bubbles $^{2}$ trap them near the region of most intense electric field.

In three dimensions, the magnetic field can have a guiding component normal to the plane. Whether it is externally applied or self consistently generated (see below), a guide field can enhance particle acceleration by keeping ions near the intense electric fields in the reconnection region. The electric field is not measured directly in our device, because the flux functions lack sufficient symmetry to compute it from magnetic probe data. However, if reconnection occurs, in the sense of global topology change, then it is axiomatic that an electric field is required along a guide field that threads the reconnection region. In fact, a viable definition of three dimensional reconnection at a point at which $\mathbf{B}$ does not vanish ${ }^{3,4}$ is the appearance of an electric field along the guide field. We observe topology change and energetic particles, so one concludes that there is a strong reconnection electric field along the separator. A guide field is also generated along the $\mathrm{X}$-line, implying a locally generated parallel electric field $\mathbf{E} \cdot \mathbf{B} \neq 0 .{ }^{3-6}$ Several models of reconnection include mechanisms for generation of out of plane fields. ${ }^{7}$

Magnetic reconnection may play a significant role in the acceleration of charged particles to ultra-relativistic energies on astrophysical scales. The key feature is that the reconnection electric field is of order $v B$ where $v$ is a typical magnetohydrodynamic (MHD) flow velocity and $B$ is a typical magnetic field strength. If magnetic reconnection plays a role in particle acceleration then maximum particle energies should scale as the reconnection electromotive force (EMF) 


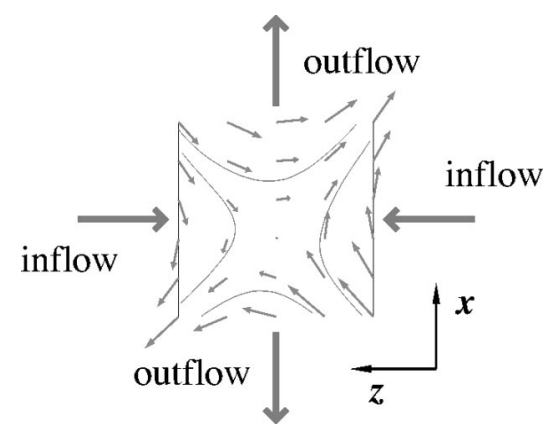

FIG. 1. Two-dimensional reconnection geometry. Inflowing magnetofluid is decoupled by reconnection. By conservation of energy, the outflow velocity is the Alfvén speed $v_{A}$. This view is actually a 2D projection of 3D SSX data.

$\mathcal{E}=\int \mathbf{E} \cdot \mathbf{d l} \cong v B L$ where $L$ is a characteristic length of the system along the electric field. ${ }^{8}$

We report a measurement of ions accelerated along the $\mathrm{X}$-line coincident with the formation of three-dimensional magnetic reconnection structures. Ion detectors are spatially aligned with the 3D structure and detection is temporally correlated with the magnetics. We have clear evidence of a component of the magnetic field $\mathbf{B}$ along the X-line (i.e., in the third dimension, normal to the classical X-point). In the terminology of Hesse and Schindler, ${ }^{4}$ this correponds to fully three-dimensional "finite B" reconnection. We have also mapped out the energy distribution of the ions and find it to be super-thermal and super-Alfvénic. Measurements agree with an accelerated ion distribution characterized by a drift energy of about $90 \mathrm{eV}$ and thermalized to $30 \mathrm{eV}$ with $E_{\max }$ $\geqslant 200 \mathrm{eV}$. In addition, test particle simulations using MHD data from SSX simulations and run with dimensionless parameters similar to the experiment $(S=1000, \beta=0.1)$ show acceleration of ions up to $2 v_{\text {Alf }}$ in a few Alfvén times.

In Sec. II, we provide astrophysical motivation for this work and review recent related experiments. In Sec. III, the Swarthmore Spheromak Experiment is described and experimental results are reported. In Sec. IV, results of numerical simulations of reconnection and particle acceleration in SSX are reported. Finally, in Sec. V future directions are discussed.

\section{ASTROPHYSICAL MOTIVATION AND PRIOR EXPERIMENTS}

There is a connection between complex magnetic structure and energetics. Simple potential fields $\boldsymbol{\nabla} \times \mathbf{B}=0$ and force-free fields $\boldsymbol{\nabla} \times \mathbf{B}=\lambda \mathbf{B}$ are in a minimum energy state and have no excess energy to give to particles. Measured away from the reconnection zone, the reconnection electric field is $\mathbf{E}=-\mathbf{v} \times \mathbf{B}$. Near the center of the reconnection zone some nonideal effect (resistivity, Hall effect, electron pressure, etc.) supports an electric field of about the same magnitude. But unless the geometry is nontrivial, e.g., if there is a component of $\mathbf{B}$ along $\mathbf{E}(\mathbf{E} \cdot \mathbf{B} \neq 0)$ it is difficult for the electric field to impart energy to the particles, ${ }^{3,4}$ because particles escape easily from a simple X-point configuration centered about a null point.
There has been significant evidence of energetic particles associated with flares from Yohkoh. ${ }^{9,10}$ Yohkoh observations show hard $\mathrm{x}$-ray sources $(>50 \mathrm{keV})$ located at the loop-tops and footpoints of flares suggesting electron acceleration along and above the loops. In addition, x-ray bright points (XBPs) have been observed at loop crossings. ${ }^{11}$ Particle acceleration by magnetic reconnection has also been considered for active galactic nuclei, ${ }^{12}$ extragalactic jets ${ }^{13}$ and accretion-induced collapsed pulsars. ${ }^{14}$ In every case, the magnetic geometry is inherently three dimensional though the analysis is greatly simplified by projecting into two dimensions. Particle acceleration in the context of solar flares has been extensively reviewed. ${ }^{15,16}$

The evidence for the existence of cosmic rays with energies in excess of $10^{20} \mathrm{eV}$ is overwhelming. Yet, energetic particles born beyond about $100 \mathrm{Mpc}$ should have been slowed by the Greisen-Zatsepin-Kúzmin (GZK) energy cutoff $\left(\sim 5 \times 10^{19} \mathrm{eV}\right) .{ }^{17,18}$ The GZK cutoff is due to inverse Compton scattering (photomeson production) off the extremely blue-shifted microwave background radiation at very high relativistic $\gamma$. The conclusion is that sources of cosmic rays with energies above the GZK cutoff must be within 100 $\mathrm{Mpc}$ of earth (and there are no pathological sources this close to the local group). Particle acceleration by threedimensional magnetic reconnection in local, large scale magnetic structures is a possible mechanism to exceed the GZK energy cutoff within $100 \mathrm{Mpc}$.

Several experiments have measured energetic particles in reconnection geometries but the emphasis has been on Alfvénic or sub-Alfvénic outflow in the 2D plane. ${ }^{19-23}$ In SSX, we directly observed an Alfvénic ion jet correlated with a reconnection event measured with a 1D probe. ${ }^{19}$ Gekelman et al. directly observed outflow at up to $0.6 c_{s}$. Their $\beta=2 \mu_{0} n k T / B^{2}$ was close to unity (about 0.5 ) so this outflow speed corresponds to about $0.4 v_{A} \cdot{ }^{20}$ Inflow speeds were about $0.3 c_{s} \cong 0.2 v_{A}$. They also observed very weak axial ion drifts $\left(v_{i} \ll 0.1 c_{s}, 0.1 v_{A}\right)$ associated with the current sheet. They used three pairs of planar one-sided collectors (Mach probes) to measure local ion flow. Interestingly, they obtained the classical MHD flow pattern with un-magnetized ions (the so-called EMHD regime). Gekelman et al. also studied the anisotropy of the electron distribution in the reconnection neutral sheet using a novel electrostatic electron energy analyzer ${ }^{24}$ and found an energetic electron tail along the X-line.

Ono et al. observed ion acceleration up to the Alfvén speed by Doppler shifts of impurity lines in an MHD plasma. ${ }^{22,23}$ Measurements were made with a polychometer along a chord that could be scanned radially. They found bi-directional outflow corresponding to the "slingshot" effect of newly reconnected flux. Peak velocities were 1.2 $\times 10^{6} \mathrm{~cm} / \mathrm{s}$ which corresponded to about the Alfvén speed in their experiment. This experimental measurement corresponds directly with reconnection driven bi-directional outflows observed on the $\operatorname{sun}^{25}$ and in the magnetosphere. ${ }^{26,27}$

Ono et al. also observed rapid ion heating (from Doppler broadening) from 10 to $200 \mathrm{eV}$ in about $10 \mu \mathrm{s}$ after the bi-directional outflow. ${ }^{22,23}$ Optical methods typically average along the line of sight making it difficult to localize the 


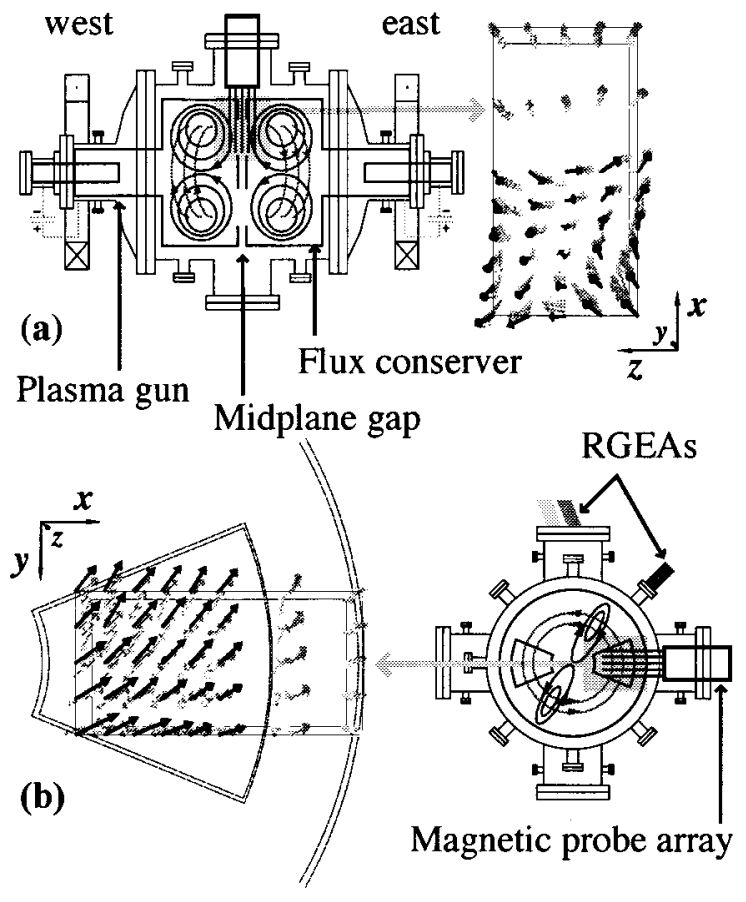

FIG. 2. Top and end views of the Swarthmore Spheromak Experiment. Spheromaks of either helicity are formed on the east and west side by magnetized plasma guns. Reconnection occurs through large slots in the back walls at the midplane. The 3D magnetic probe array and retarding grid energy analysers (RGEAs) are located at the midplane. Magnifield views illustrate magnetic probe locations and data shown as arrows (the longest arrows are approximately $800 \mathrm{G}$ ). The view in (a) is the $\hat{\mathbf{x}}-\hat{\mathbf{z}}$ plane, the view in (b) is the $\hat{\mathbf{x}}-\hat{\mathbf{y}}$ plane. Of the $200 \mathbf{B}$ vectors, only those on facing planes are highlighted.

source of heating/acceleration. In addition, a radial line of sight mixes toroidal and poloidal flow components. Recently however, Hsu et al. used a local optical probe technique to measure heating of the majority ions in the reconnection layer of Magnetic Reconnection Experiment (MRX) ${ }^{28,29} \mathrm{He}$ observed a local increase in $T_{i}$ of about a factor of 3 (from 5 to $15 \mathrm{eV}$ ). The line of sight was $5 \mathrm{~cm}$ for those measurements. Hsu et al. also measured downstream outflow $\left(v_{i}\right.$ $\cong 0.2 v_{A}$ ) and found plasma rotation outside the layer but low ion velocity along the $\mathrm{X}$-line $\left(v_{i} \ll v_{t h}\right)$.

\section{SSX EXPERIMENTS}

The Swarthmore Spheromak Experiment $(\mathrm{SSX})^{1}$ is designed to study magnetic reconnection and particle acceleration due to the controlled, partial merging of two spheromaks (see Fig. 2). We are able to generate spheromaks of either sign of magnetic helicity (right-handed or left-handed twist) on either side of the experiment (east or west). SSX is unique in that the spheromaks are generated by plasma guns away from the reconnection zone. ${ }^{30}$ Neutral gas and vacuum magnetic fields are introduced at the guns but only fully ionized plasma and imbedded magnetic flux convect into the reconnection zone. In addition, SSX employs a segmented copper boundary at the midplane to allow partial merging as well as allowing each spheromak to relax to its own equilibrium configuration. The linear dimensions of the open chevronshaped segments are large enough to allow significant inter-

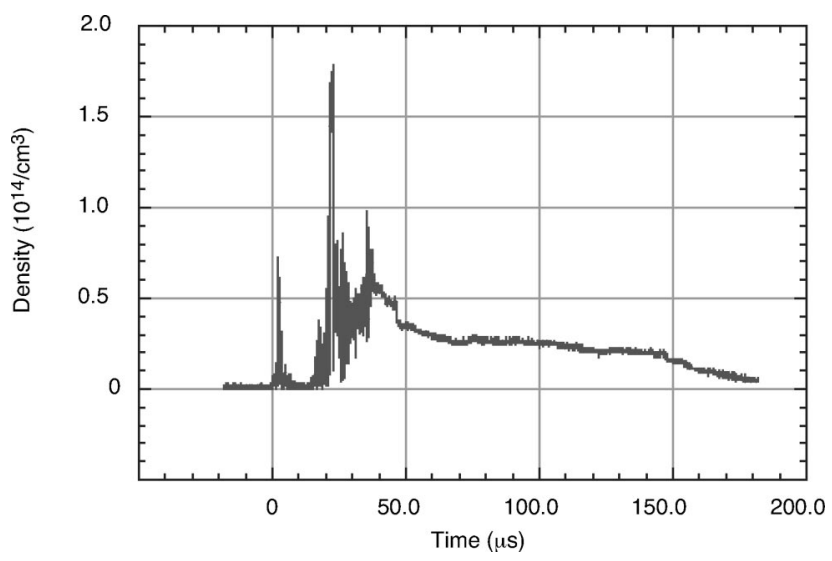

FIG. 3. Density trace from quadrature $\mathrm{He}-\mathrm{Ne}$ interferometer.

action (about the spheromak minor radius $a=0.13 \mathrm{~m}$ ). The segmented boundary also removes axisymmetry, making the experiment fully and inherently three-dimensional.

Early in the discharge we are able to study driven reconnection as the still forming spheromaks merge at the midplane at close to the Alfvén speed. ${ }^{19}$ Later in the discharge, the two spheromaks separately relax to nearly force-free equilibria ${ }^{30}$ so that we can study spontaneous reconnection along the interface between them. Energetic particles and soft $\mathrm{x}$-rays traverse the field-free, high vacuum gap between flux conservers to various detectors on the midplane. Special ports have been designed to view the reconnection region through a small solid angle along the $\mathrm{X}$-line so we have good spatial localization of the reconnection region. Diagnostic attention can be focussed on those regions where reconnection is known to occur.

Large scale features of SSX plasmas are in the magnetohydrodynamic (MHD) regime $\left(S \gg 1, \rho_{i} / L \ll 1, v_{A} / c\right.$ $\ll 1)$. We measure electron density with a quadrature $\mathrm{He}-\mathrm{Ne}$ interferometer system. Typical SSX line-averaged densities are about $n_{e}=5 \times 10^{13} \mathrm{~cm}^{-3}$ (see Fig. 3) so $c / \omega_{p i} \cong 2$ $-3 \mathrm{~cm}$. We measure local electron density and temperature with a triple Langmuir probe $\left(T_{e} \cong 20 \mathrm{eV}\right)$. The ion temperature is estimated from ion energy analysers $\left(T_{i} \cong 20 \mathrm{eV}\right)$. Mean magnetic fields in SSX are about $0.05 \mathrm{~T}$ so $v_{A}$ $=B / \sqrt{\mu_{0} \rho} \cong 10^{5} \mathrm{~m} / \mathrm{s}$ and $\rho_{i} \leqslant 1 \mathrm{~cm}$. The Lundquist number is given by $S=\mu_{0} \sigma v_{A} L \leqslant 1000$. The Spitzer conductivity at $20 \mathrm{eV}$ is $\sigma_{S p}=1.7 \times 10^{5} \Omega^{-1} \mathrm{~m}^{-1}$. The electron mean free path is $\geqslant 0.1 \mathrm{~m}$.

We measure 200 B vectors on a $5 \times 5 \times 8$ threedimensional grid using a multiplexer technique to perform the 600 measurements at sub- $\mu s$ resolution in time and $2 \mathrm{~cm}$ resolution in space (see Fig. 2). ${ }^{6,31}$ Data are obtained in a single shot. We then interpolate field lines on the 3D data using fourth order Runga-Kutta integration. Field lines are integrals of

$$
\frac{d x_{i}}{d s}=\frac{B_{i}}{B},
$$

where $d s$ is an element of arc length and $B$ is the magnitude of the field $\left(B_{i} / B\right.$ is the component of the unit vector along $\left.B_{i}\right)$. In every case presented here, the curvature of the field 


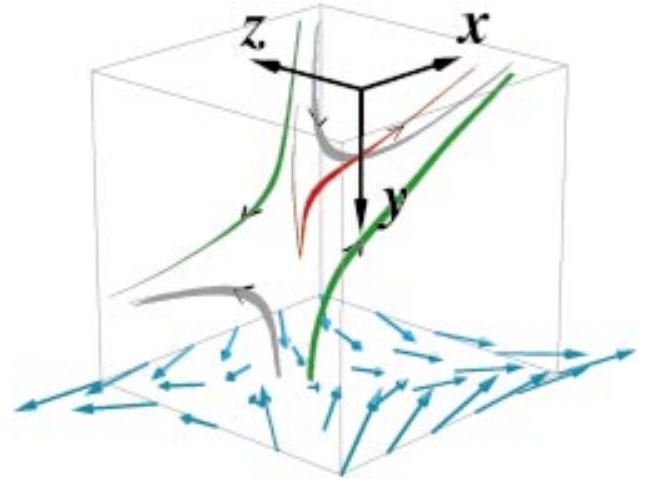

FIG. 4. (Color) Three-dimensional reconnection. Field lines integrated through the 3D data set viewed approximately in the $(\hat{\mathbf{x}}, \hat{\mathbf{y}}, \hat{\mathbf{z}})=(1,0,1)$ direction show a vestige of the $\mathrm{X}$-geometry with a field line near the $\mathrm{X}$-point that is both swept and sheared (red). Inflowing lines are green, outflowing lines are gray. The magnetic vector data is suppressed except for the lower plane for clarity. This is during the relaxation phase at time $t=64 \mu \mathrm{s}$ from a single shot.

lines is large compared to the probe separation ( $r_{\text {line }}$ $\left.\geqslant \delta_{\text {probe }}\right)$ so we have confidence in the accuracy of the integration. We can compute current density $\mathbf{J}=\boldsymbol{\nabla} \times \mathbf{B} / \mu_{0}$ and magnetic energy density $W=\int B^{2} / 2 \mu_{0} d^{3} x$ from the data. Calibration is performed using precise Helmholtz coils and known line currents.

In Fig. 4 we present a view of the measured threedimensional structure of the magnetic reconnection geometry
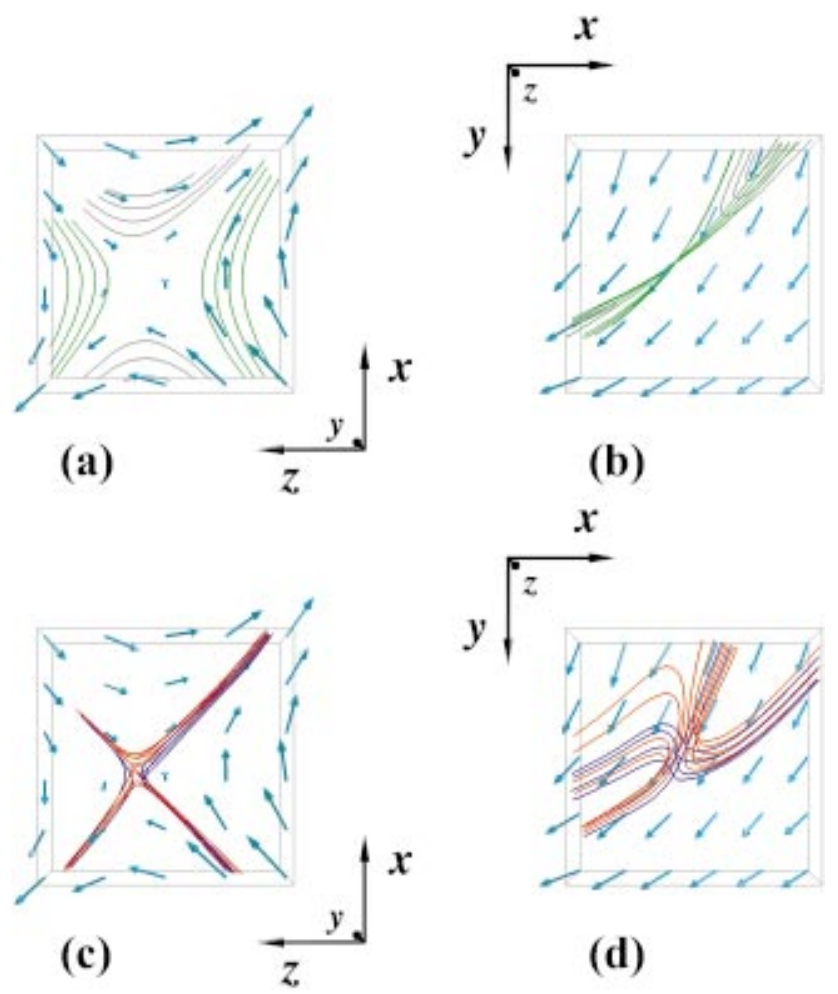

FIG. 5. (Color) Outer and inner reconnection regions. Field lines integrated in the outer $(a, b)$ and inner $(c, d)$ reconnection regions viewed in the $\hat{\mathbf{y}}$ (a) and $\hat{\mathbf{z}}$ (b) directions. The $\hat{\mathbf{y}}$ view shows the X-geometry while the $\hat{\mathbf{z}}$ view shows the swept/sheared structure particularly in the inner reconnection region (d). The magnetic vector data is displayed here for the middle $\hat{\mathbf{x}}-\hat{\mathbf{z}}$ plane (a, c) and the rear $\hat{\mathbf{x}}-\hat{\mathbf{y}}$ plane $(\mathrm{b}, \mathrm{d})$. This is a 40 shot average.
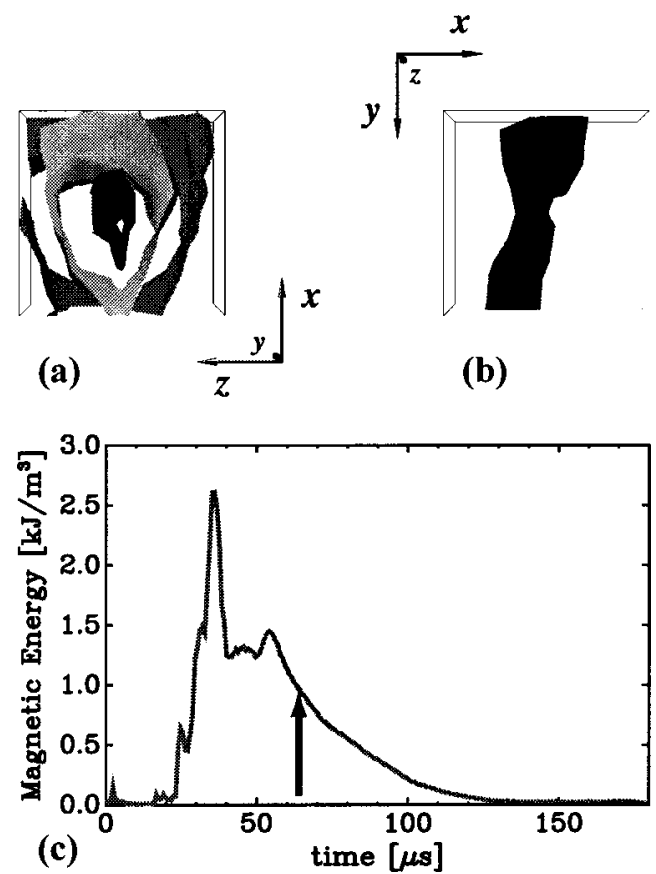

FIG. 6. Magnetic energy. (a) Plot of isosurfaces of magnetic energy using the data from Fig. 4 at the same time $t=64 \mu \mathrm{s}$. (b) Another view of the inner isosurface. (c) The time history of the average magnetic energy integrated over the volume $\int B^{2} / 2 \mu_{0} d^{3} x$.

in SSX. We show four field lines integrated through the data which reveal a vestige of the classic X-point geometry (inflow region is green, outflow gray). A fifth field line (red) integrated near the reconnection region shows what we call a "swept/sheared" structure. This is a reconnected field line that threads from the west (far) to the east (near) spheromak with $\mathbf{E} \cdot \mathbf{B}>0$. The field line is dynamically swept into the reconnection region showing a clear signature of 3D reconnection (i.e., finite $\mathbf{E} \cdot \mathbf{B}$ ). This is a counter-helicity merging shot with the east spheromak left-handed and the west spheromak right-handed. In this view, the right side of the figure is radially out of the machine. Locally, $\mathbf{B}$ is directed up and to the right on the east (near) side and down and to the left on the west (far) side. The time is well into the relaxation phase $t=64 \mu \mathrm{s}$. We have suppressed the magnetic vector data (except for the lower plane) for clarity.

In Fig. 5, we compare the geometry of field lines integrated both in the inner and outer reconnection regions. The data presented here is from a 40 shot average and we display magnetic vector data in only a $5 \times 5 \times 6$ portion of the array. The reconnection region is viewed in the $\hat{\mathbf{y}}$ (left) and $\hat{\mathbf{z}}$ (right) directions. In the top panels $(\mathrm{a}, \mathrm{b})$, field lines are integrated about one grid spacing (about $c / \omega_{p i}$ ) outside the $\mathrm{X}$-line (green inflow, gray outflow). We observe the classic $\mathrm{X}$-structure (a) that seems to lie on a curved surface (b). In the bottom panels $(\mathrm{c}, \mathrm{d})$, field lines are integrated near the $\mathrm{X}$-line (blue inflow, red outflow). We observe a tighter $\mathrm{X}$-structure (c) which is dynamically swept along the X-line and is highly twisted and sheared (d).

In Fig. 6, we display the 3D structure using magnetic energy. In Figs. 6(a) and 6(b), we show some isosurfaces of magnetic energy density $B^{2} / 2 \mu_{0}$ for the same single shot and 


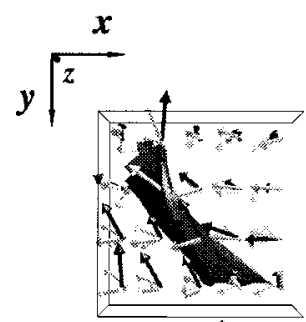

(a)

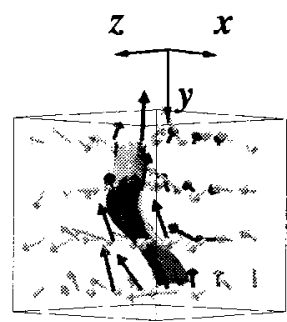

(b)

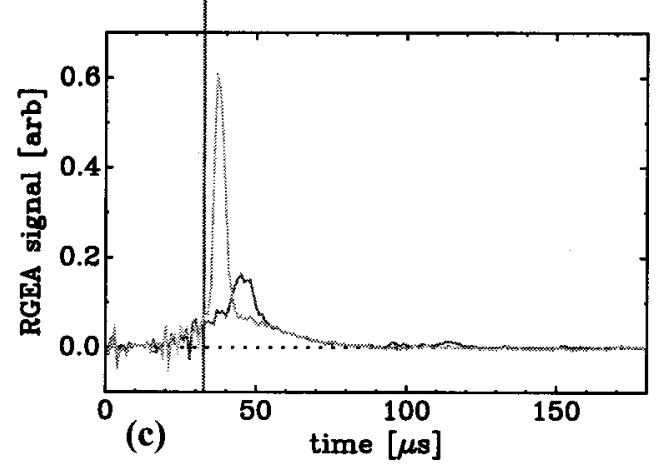

FIG. 7. Current sheet and ion pulses. (a, b) Plot of the current density from Fig. $4\left(J=\nabla \times B / \mu_{0}\right)$ but during the early, driven reconnection phase $t$ $=32 \mu \mathrm{s}$ (two views). The peak current density is about $3 \mathrm{MA} / \mathrm{m}^{2}$. (c) Ion pulses measured by our retarding grid energy analyzers (RGEA). The early pulse is from the RGEA aligned with the reconnection electric field. The later pulse is from the RGEA aligned with the Alfvénic outflow.

at the same time as in Fig. $4(t=64 \mu \mathrm{s})$. We suppress the magnetic vector data for this plot. Note that there is a magnetic energy minimum roughly aligned with the $\mathrm{X}$-line (as one would expect). In Fig. 6(c), we present a time history of the average magnetic energy integrated over the volume of measurement $\int B^{2} / 2 \mu_{0} d^{3} x$. The arrow indicates the time displayed in (a) and (b).

In Figs. 7(a) and 7(b), we present a plot of the current density from the same single shot as in Fig. 4 ( $\mathbf{J}$ $\left.=\nabla \times \mathbf{B} / \mu_{0}\right)$ but during the early, driven reconnection phase $t=32 \mu \mathrm{s}$. For clarity, we highlight $\boldsymbol{\nabla} \times \mathbf{B}$ near the reconnection zone and integrate lines of $\mathbf{J}$ to display a current channel. Note that during the driven phase, the current peaks and is directed towards the top of the box (roughly the $-\hat{\mathbf{y}}$ direction). Since the inflow velocity has stagnated in the layer, we believe $\mathbf{J} \| \mathbf{E}$ there, so this is also the direction of the local electric field. It is along this direction that we have placed one of our retarding grid energy analyzers (RGEAs). In Fig. 7(c), we show ion pulses measured by two of our RGEAs: one aligned with the reconnection electric field (normal to the local reconnection layer) and another aligned with the Alfvénic outflow (across the reconnection layer). The early pulse is from the RGEA aligned with the reconnection electric field. Note that the time displayed for Figs. 7(a) and 7(b) is just before the peak of the ion pulse of this detector demonstrating temporal correlation between the peak of the reconnection electric field and the peak of the pulse of ions flowing in that direction. The later pulse is from the RGEA aligned with the slower Alfvénic outflow.

We are able to scan the gate voltage of the RGEAs from shot to shot. In Fig. 8, we show five pulses from the RGEA
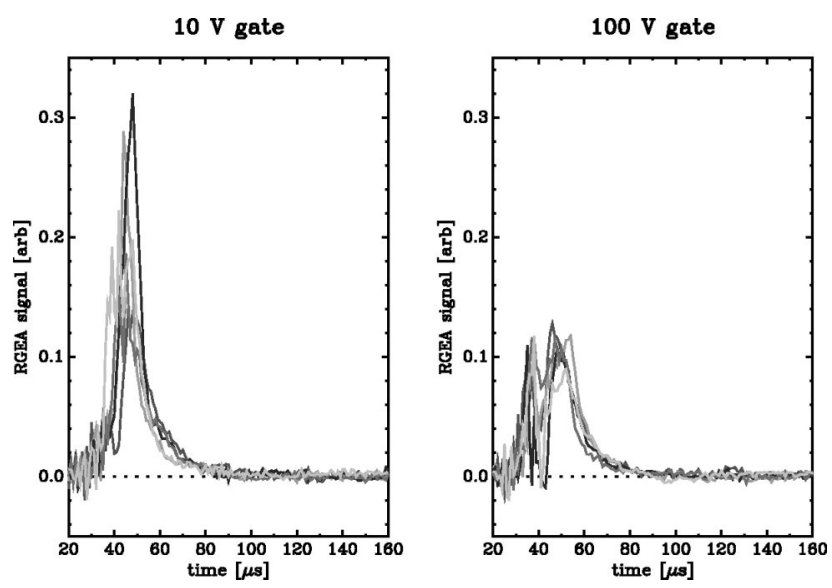

FIG. 8. Ion pulses from the RGEA aligned with the reconnection electric field at varying gate voltage, $10 \mathrm{~V}$ (left) and $100 \mathrm{~V}$ (right)

aligned with the reconnection electric field with the ion discrimination gate set at $10 \mathrm{~V}$ (left) and $100 \mathrm{~V}$ (right). Each trace was obtained from shots under identical conditions. Note that a slow population of ions is retarded by the $100 \mathrm{~V}$ gate but there is clear evidence of a large population of ions with energies exceeding $100 \mathrm{~V}$. These are super-Alfvénic protons in SSX. In Fig. 9, we present the measured ion distribution as a function of gate voltage. We show the mean and standard deviation $\sigma$ for 10 shots at each voltage and fit the data with a three parameter model (amplitude, ion temperature and ion drift energy). To improve signal to noise on the RGEAs, the 3D magnetic array was removed to obtain this large data set. The best fit (solid curve) is characterized by a drift energy of about $90 \mathrm{eV}$ and thermalized to $30 \mathrm{eV}$. The dashed lines show $\pm \sigma$ from the best fit. Note that the distribution is clearly super-thermal in contrast to earlier work. ${ }^{20,28}$

\section{SSX SIMULATIONS}

Numerical simulations of magnetic reconnection and subsequent particle acceleration have been performed in both two-dimensions ${ }^{32,33,2}$ and three-dimensions. ${ }^{34,35}$ Nonrelativ-

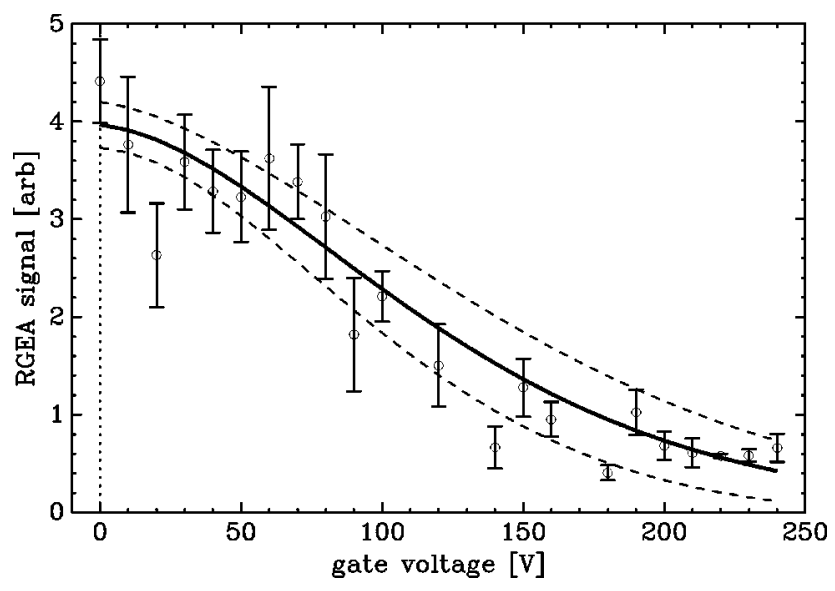

FIG. 9. Energy distribution. These measurements agree with an accelerated ion distribution characterized by a super-thermal, super-Alfvénic drift energy of about $90 \mathrm{eV}$ and thermalized to $30 \mathrm{eV}$. 

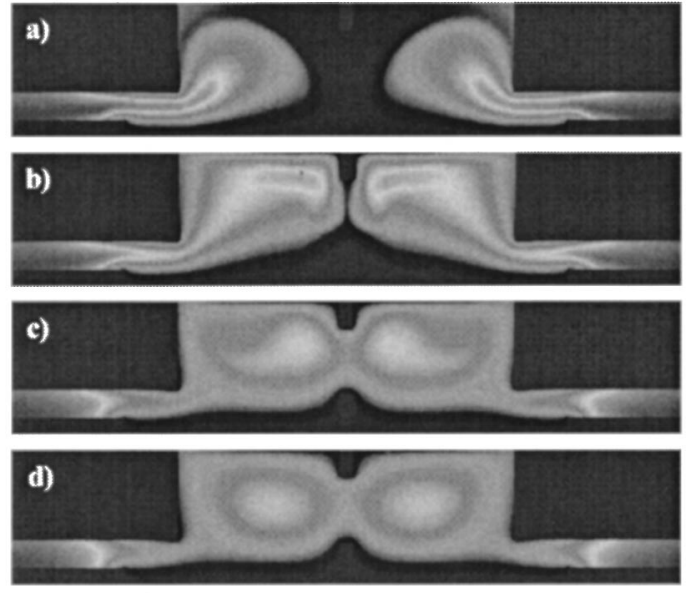

FIG. 10. Evolution of magnetic flux from TRIM simulation. (a) Formation $t=-2.7 \mu \mathrm{s}$, (b) merging $t=0 \mu \mathrm{s}$, (c) relaxation $t=13.5 \mu \mathrm{s}$, (d) equilibrium $t=23.4 \mu \mathrm{s}$.

istic neutral point acceleration was considered in the context of a planar sheet pinch in the presence of finite amplitude fluctuations. $^{32,33,2}$ Recent work is fully relativistic and has included the effects of losses due to synchrotron radiation and inverse Compton scattering. ${ }^{34}$

We have performed numerical simulations of SSX reconnection experiments using a 2-1/2 D cylindrically symmetric nonideal single fluid MHD code called TRIM $^{36}$ to simulate the time evolution of the electrodynamic fields, momentum density and plasma density. We demonstrated that an $a b$ initio calculation (i.e., from gun formation to equilibrium using SSX boundary conditions) reproduced our experimentally measured global profiles well. ${ }^{30,37}$ Reversal of the magnetic field and current filamentation occur at the reconnection layer both in the experiment and in the simulation. The principal difference is that the current sheet thickness is determined by Ohmic dissipation in the single fluid MHD TRIM simulation while two-fluid effects limit the current sheet thickness to $c / \omega_{p i}$ in the SSX experiment. ${ }^{19,37}$ Finally, we have performed test particle simulations using MHD data from TRIM and run with dimensionless parameters similar to that of the experiment $(S=1000, \beta=0.1)$.

In Fig. 10, we present plots of the numerically calculated poloidal flux $\left(\Phi_{\mathrm{pol}}=r A_{\phi}\right)$ at several key times. In Fig. 10(a) $(t=-2.7 \mu \mathrm{s})$ magnetofluid has been ejected out of the coaxial magnetized plasma guns and moves towards the reconnection zone at close to $v_{A}$. In Fig. 10(b), initial merging has commenced. We define the simulation $t=0 \mu \mathrm{s}$ at this moment which corresponds approximately to the experimental time depicted in Fig. 7. By $t=13.5 \mu \mathrm{s}$, significant reconnection has occurred and the system has begun to relax. By $t$ $=23.4 \mu \mathrm{s}$, an equilibrium has been established and resistive decay has begun.

Numerical experiments were performed in which the trajectories of $10^{5}$ protons are calculated using the dynamical MHD data from TRIM. The test particle simulation is run with dimensionless parameters similar to the experiment, and particles are permitted to escape when they encounter the simulated SSX boundaries. ${ }^{38}$ The initial condition considered
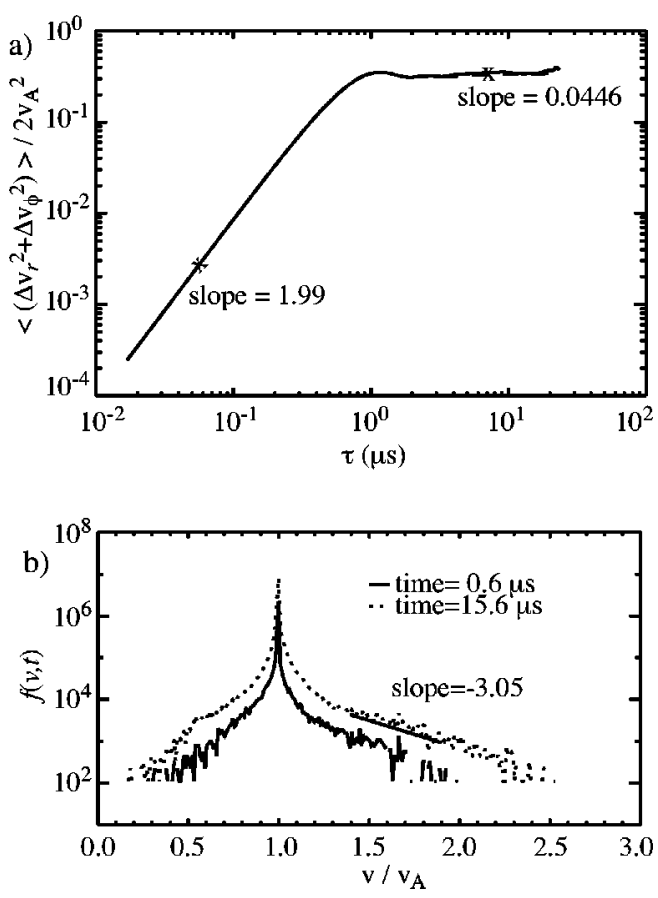

FIG. 11. Mean square velocity increment and tail formation. (a) Direct acceleration of protons for short times. (b) Distribution function $f(v, t)$ of escaping protons at $t=0.6 \mu \mathrm{s}$ and $t=15.6 \mu \mathrm{s}$ shows formation of a superAlfvénic tail.

is that all protons are injected into the reconnection region at $v_{A}$ but with random positions and gyrophases. MHD activity related to reconnection is responsible for accelerating charged particles. The process includes two phases-a strong but short duration direct acceleration in the quasi-steady reconnection electric field, and a weaker longer lived stochastic component associated with turbulence.

Here we present two diagnostics that illustrate the statistical behavior of the energized particles in the simulations. Further detail can be found in the references. ${ }^{37,38}$ In Fig. 11(a), we show the mean-square velocity increment versus the time of separation. In other words, for each particle, we compute the square of the change in velocity in the toroidal plane (roughly in the direction of the reconnection electric field) for a specified time separation $\tau$ :

$$
\Delta v_{r}(t, \tau)^{2}+\Delta v_{\theta}(t, \tau)^{2}
$$

then average over all times $t$ and all particles and finally normalize to $2 v_{A}^{2}$. The early time response is distinctive-we see that for time increments less than about an Alfvén time $(1 \mu \mathrm{s})$, the proton velocity increases linearly with time separation $\tau$ regardless of discharge time $t$. At later times, the velocity changes are random. If the velocity changes were diffusive, we would expect the mean-square velocity increment to grow linearly with time (i.e., $\Delta v$ $\left.\propto \tau^{1 / 2}\right)$. After about $1 \mu$ s the acceleration is slower than that so we call it sub-diffusive. In Fig. 11(b), we plot the distribution function of protons $f(v, t)$ that escape the simulation domain. We see that at early times $(t=0.6 \mu \mathrm{s})$ a tail is beginning to form and by several Alfvén times well into the formation phase $(t=15.6 \mu \mathrm{s})$ a significant tail of ions with 

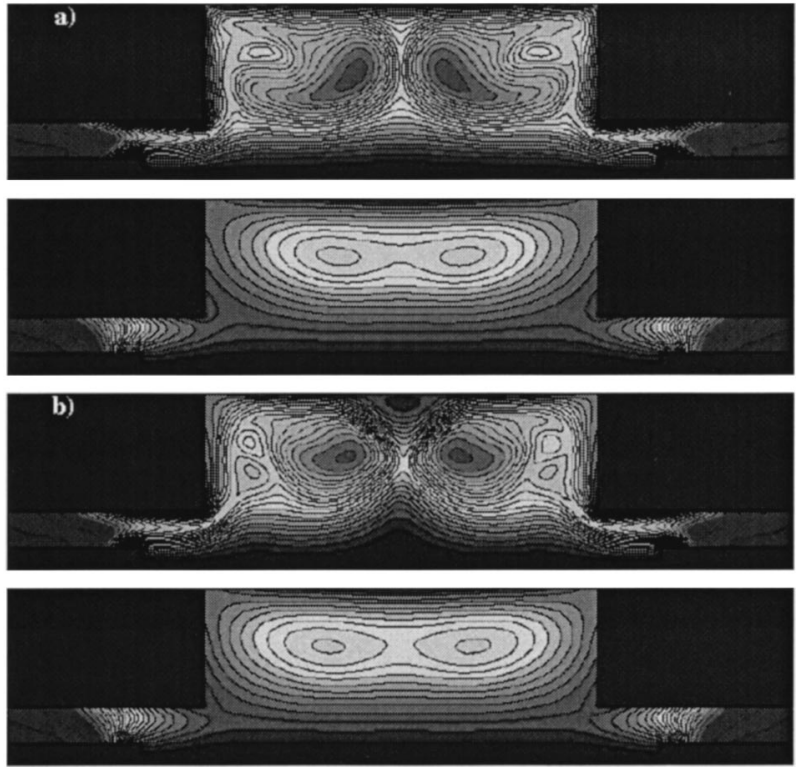

FIG. 12. Doublet CT. Full merging of SSX spheromaks will yield a new object with some remaining toroidal flux called a doublet CT. (a) Isothermal equation of state, reconnection layer has a Y-point geometry and is very thin at $t=2 \tau_{\text {Alf }}$ (above), evolution to an FRC equilibrium proceeds at later times ( $t=55 \tau_{\text {Alf }}$ ) (below). (b) Polytropic equation of state $\gamma=5 / 3$, reconnection layer has an X-point geometry and is broad at $t=2 \tau_{\text {Alf }}$ (above), evolution to an FRC equilibrium proceeds at later times ( $t=55 \tau_{\text {Alf }}$, below) very much like the isothermal case.

$v>2 v_{A}$ has been accumulated. Note also that the distribution, originally a $\delta$-function at $v_{A}$ has broadened substantially.

\section{FUTURE DIRECTIONS AND SUMMARY}

Finally, we have performed TRIM simulations of full merging of SSX spheromaks anticipating future experiments. In the modified experiment (called SSX-FRC) we will remove the wall between the spheromaks making the configuration axisymmetric. We have employed a variety of equations of state then compared reconnection and merging results. New results indicate that the width of the reconnection layer is increased and the rate of reconnection is slowed by employing a polytropic equation of state (see Fig. 12). In the isothermal case [Fig. 12(a)], a distinctive reconnection Y-point is formed early in the driven phase of merging $(t$ $\left.=2 \tau_{\text {Alf }}\right)$, while in the polytropic case $\left(P V^{\gamma}=\right.$ const with $\gamma$ $=5 / 3)$, the reconnection layer is broader and forms an $\mathrm{X}$-point [Fig. 12(b)]. Late in the decay phase $\left(t=55 \tau_{\text {Alf }}\right)$, both cases relax to near FRC equilibria (which we call a doublet CT).

To summarize, we have reported a measurement of ions accelerated along the $\mathrm{X}$-line coincident with the formation of three-dimensional magnetic reconnection structures. RGEAs specially designed to align with the $3 \mathrm{D}$ structures show ion pulses temporally correlated with the magnetics. We have clear evidence of a component of the magnetic field $\mathbf{B}$ swept and sheared along the $\mathrm{X}$-line demonstrating the full 3D aspect of reconnection. We have also mapped out the energy distribution of the ions accelerated by these magnetic structures and find it to be both super-thermal and super-Alfvénic.
Measurements agree with an accelerated ion distribution characterized by a drift energy of about $90 \mathrm{eV}$ and thermalized to $30 \mathrm{eV}$ with $E_{\max } \geqslant 200 \mathrm{eV}$.

\section{ACKNOWLEDGMENTS}

Discussions with Paul Bellan (Caltech), Stan Owocki (Bartol), Mike Schaffer (GA), Tom Kornack (PPPL), John Boccio, Phil Everson and Jean Griffin (Swarthmore) are greatfully acknowledged. M.L. was supported in part by DVSGC. Equipment assistance by Steve Palmer.

This work was performed under Department of Energy (DOE) Grants No. DE-FG02-00ER54604 and No. ER54490.

${ }^{1}$ M. R. Brown, Phys. Plasmas 6, 1717 (1999).

${ }^{2}$ J. J. Ambrosiano, W. H. Matthaeus, M. L. Goldstein, and D. Plante, J. Geophys. Res. 93, 14383 (1988).

${ }^{3}$ K. Schindler, M. Hesse, and J. Birn, J. Geophys. Res. 93, 5547 (1988).

${ }^{4}$ M. Hesse and K. Schindler, J. Geophys. Res. 93, 5559 (1988).

${ }^{5}$ E. R. Priest and T. G. Forbes, Magnetic Reconnection (Cambridge University Press, Cambridge, 2000).

${ }^{6}$ C. D. Cothran, M. Landreman, W. H. Matthaeus, and M. R. Brown, Phys. Rev. Lett. (submitted).

${ }^{7}$ M. A. Shay, J. F. Drake, R. E. Denton, and D. Biskamp, J. Geophys. Res. 103, 9165 (1998).

${ }^{8}$ K. Makishima, Astron. Nachr. 320, 163 (1999).

${ }^{9}$ S. Masuda, T. Kosugi, H. Hara, S. Tsuneta, and Y. Ogawara, Nature (London) 371, 495 (1994).

${ }^{10}$ S. Tsuneta, Publ. Astron. Soc. Jpn. 47, 691 (1995).

${ }^{11}$ L. van Driel-Gesztelyi, B. Schmieder, G. Cauzzi, N. Mein, A. Hofmann, N. Nitta, H. Kurokawa, P. Mein, and J. Staiger, Sol. Phys. 163, 145 (1996).

${ }^{12}$ R. Schopper, H. Lesch, and G. T. Birk, Astron. Astrophys. 335, 26 (1998).

${ }^{13}$ Y. E. Litvinenko, Astron. Astrophys. 349, 685 (1999).

${ }^{14}$ E. M. G. Dal Pino and A. Lazarian, Astrophys. J. Lett. 536, L31 (2000).

${ }^{15}$ J. A. Miller, P. J. Cargill, A. G. Emslie, G. D. Holman, B. R. Dennis, T. N. LaRosa, R. M. Winglee, S. G. Benka, and S. Tsuneta, J. Geophys. Res. 102, 14631 (1997).

${ }^{16}$ H. Hudson and J. Ryan, Annu. Rev. Astron. Astrophys. 33, 239 (1995).

${ }^{17}$ K. Greisen, Phys. Rev. Lett. 16, 748 (1966).

${ }^{18}$ J. W. Cronin, Rev. Mod. Phys. 71, S165 (1999).

${ }^{19}$ T. W. Kornack, P. K. Sollins, and M. R. Brown, Phys. Rev. E 58, R36 (1998).

${ }^{20}$ W. Gekelman, R. L. Stenzel, and N. Wild, J. Geophys. Res. 87, 101 (1982).

${ }^{21}$ R. L. Stenzel, W. Gekelman, and N. Wild, J. Geophys. Res. 87, 111 (1982).

${ }^{22}$ Y. Ono, M. Yamada, T. Akao, T. Tajima, and R. Matsumoto, Phys. Rev. Lett. 76, 3328 (1996).

${ }^{23}$ Y. Ono, M. Inomoto, T. Okazaki, and Y. Ueda, Phys. Plasmas 4, 1953 (1997).

${ }^{24}$ W. Gekelman and R. L. Stenzel, Phys. Rev. Lett. 54, 2414 (1985).

${ }^{25}$ D. E. Innes, B. Inhester, W. I. Axford, and K. Wilhelm, Nature (London) 386, 811 (1997).

${ }^{26}$ T. D. Phan et al., Nature (London) 404, 848 (2000).

${ }^{27}$ M. Oieroset, T. D. Phan, M. Fujimoto, R. P. Lin, and R. P. Lepping, Nature (London) 412, 414 (2001)

${ }^{28}$ S. C. Hsu, G. Fiksel, T. A. Carter, H. Ji, R. M. Kulsrud, and M. Yamada, Phys. Rev. Lett. 84, 3859 (2000).

${ }^{29}$ S. C. Hsu, T. A. Carter, G. Fiksel, H. Ji, R. M. Kulsrud, and M. Yamada, Phys. Plasmas 8, 1916 (2001).

${ }^{30}$ C. G. R. Geddes, T. W. Kornack, and M. R. Brown, Phys. Plasmas 5, 1027 (1998).

${ }^{31}$ M. Landreman, C. D. Cothran, M. Kostora, J. Slough, and M. R. Brown, Rev. Sci. Instrum. (submitted).

${ }^{32}$ W. H. Matthaeus, J. J. Ambrosiano, and M. L. Goldstein, Phys. Rev. Lett. 53, 1449 (1984).

${ }^{33}$ W. H. Matthaeus and S. L. Lamkin, Phys. Fluids 29, 2513 (1986). 
${ }^{34}$ R. Schopper, G. T. Birk, and H. Lesch, Phys. Plasmas 6, 4318 (1999).

${ }^{35}$ T. Kobak and M. Ostrowski, Mon. Not. R. Astron. Soc. 317, 973 (2000).

${ }^{36}$ D. Schnack, I. Lottati, Z. Mikic, and P. Satyanarayana, J. Comput. Phys. 140, 71 (1998).
${ }^{37}$ V. S. Lukin, G. Qin, W. H. Matthaeus, and M. R. Brown, Phys. Plasmas 8, 1600 (2001).

${ }^{38}$ G. Qin, V. S. Lukin, C. D. Cothran, M. R. Brown, and W. H. Matthaeus, Phys. Plasmas 8, 4816 (2001). 